\title{
Graphs with Given Degree Sequence and Maximal Spectral Radius
}

\author{
Türker Bıyıkoğlu \\ Department of Mathematics \\ Işık University \\ Şile 34980, Istanbul, Turkey \\ turker.biyikoglu@isikun.edu.tr \\ Josef Leydold \\ Department of Statistics and Mathematics \\ University of Economics and Business Administration \\ Augasse 2-6, A-1090 Wien, Austria \\ josef.leydold@wu-wien.ac.at
}

Submitted: Jul 6, 2007; Accepted: Sep 7, 2008; Published: Sep 15, 2008

Mathematics Subject Classification: 05C35, 05C75, 05C05

\begin{abstract}
We describe the structure of those graphs that have largest spectral radius in the class of all connected graphs with a given degree sequence. We show that in such a graph the degree sequence is non-increasing with respect to an ordering of the vertices induced by breadth-first search. For trees the resulting structure is uniquely determined up to isomorphism. We also show that the largest spectral radius in such classes of trees is strictly monotone with respect to majorization.

Keywords: adjacency matrix, eigenvectors, spectral radius, degree sequence, Perron vector, tree, majorization
\end{abstract}

\section{Introduction}

Let $G(V, E)$ be a simple finite undirected graph with vertex set $V(G)$ and edge set $E(G)$. The eigenvalue of $G$ are the eigenvalues of the adjacency matrix $A(G)$. The spectral radius of $G$ is the largest eigenvalue of $A(G)$, also called the index of the graph. When $G$ is connected, $A(G)$ is irreducible and by the Perron-Frobenius Theorem (see e.g. [8]) the largest eigenvalue $\lambda(G)$ of $G$ is simple and there is a unique positive unit eigenvector. We refer to such an eigenvector $f$ as the Perron vector of $G$.

There exists a vast literature that provides upper and lower bounds on the largest eigenvalue of $G$ given some information about the graph, for previous results see [5]. 
Many recent results use the maximum, minimum or average degrees, e.g., [10, 13]. Some new results are based on the entire degree sequence, e.g., [15].

The goal of this article is slightly shifted. We want to characterize connected graphs $G$ that have greatest spectral radius in the class of all graphs with a given degree sequence. We show that in such a graph the degree sequence is non-increasing with respect to an ordering of the vertices induced by breadth-first search. (Recently similar results have been shown for the special cases of caterpillars [16] and cycles with spikes [1].) We also show that the greatest maximum eigenvalue in such classes of trees is strictly monotone with respect to some partial ordering of degree sequences. The results are related to the (partly open) problem of finding connected graphs of maximal spectral radius with given number of vertices and edges (but arbitrary degree sequences). Brualdi and Solheid [4] have shown that such graphs have stepwise adjacency matrix. We refer the reader to 6 , Sect. 3.5] for details and further discussion of this and related problems.

The paper is organized as follows: The results of this paper are stated in Section 2. In Section 3 we prove these theorems by means of a technique of rearranging graphs which has been developed in [2] for the problem of minimizing the first Dirichlet eigenvalue within a class of trees. Indeed, we will discuss the close relationship between this problem and the problem of finding trees with greatest maximum eigenvalue in Section 4.

\section{Degree Sequences and Largest Eigenvalue}

Let $d(v)$ denote the degree of vertex $v$. We call a vertex $v$ with $d(v)=1$ a pendant vertex of the graph (and leaf in case of a tree). In the following $n$ denotes the total number of vertices, i.e., $n=|V|$. A sequence $\pi=\left(d_{0}, \ldots, d_{n-1}\right)$ of nonnegative integers is called degree sequence if there exists a graph $G$ with $n$ vertices for which $d_{0}, \ldots, d_{n-1}$ are the degrees of its vertices, see Melnikov et al. [11] for relevant information. In the entire article we enumerate the degrees in non-increasing order.

We introduce the following class for which we can provide optimal results for the greatest maximum eigenvalue.

$$
\mathcal{C}_{\pi}=\{G \text { is a connected graph with degree sequence } \pi\} \text {. }
$$

For the characterization of graphs that have greatest maximum eigenvalue among all graphs in $\mathcal{C}_{\pi}$ we introduce an ordering of the vertices $v_{0}, \ldots, v_{n-1}$ of a graph by means of breadth-first search: Select a vertex $v_{0} \in G$ and create a sorted list of vertices beginning with $v_{0}$; append all neighbors $v_{1}, \ldots, v_{d\left(v_{0}\right)}$ of $v_{0}$ sorted by decreasing degrees; then append all neighbors of $v_{1}$ that are not already in this list; continue recursively with $v_{2}, v_{3}, \ldots$ until all vertices of $G$ are processed. In this way we build layers where each vertex $v$ in layer $i$ has distance $i$ from root $v_{0}$ which we call its height $h(v)=\operatorname{dist}\left(v, v_{0}\right)$. Moreover, $v$ is adjacent to some vertices $w$ in layer $i-1$. We call the least one (in the above breadthfirst search) the parent of $v$ and $v$ a child of $w$. Notice that one can draw these layers on circles. Hence we call such an ordering spiral like ordering, see [12]. 
Definition 1 (BFD-ordering). Let $G(V, E)$ be a connected graph with root $v_{0}$. Then a well-ordering $\prec$ of the vertices is called breadth-first search ordering with decreasing degrees ( $B F D$-ordering for short) if the following holds for all vertices $v, w \in V$ :

(B1) if $w_{1} \prec w_{2}$ then $v_{1} \prec v_{2}$ for all children $v_{1}$ of $w_{1}$ and $v_{2}$ of $w_{2}$, resp.;

(B2) if $v \prec u$, then $d(v) \geq d(u)$.

We call a connected graph that has a BFD-ordering of its vertices a BFD-graph.

Every graph has for each of its vertices $v$ an ordering with root $v$ that satisfies (B1). This can be found by a breadth-first search as described above. However, not all graphs have an ordering that satisfies (B2); consider the complete bipartite graph $K_{2,3}$.

Theorem 1. Let $G$ have greatest maximum eigenvalue in class $\mathcal{C}_{\pi}$. Then there exists a BFD-ordering of $V(G)$ that is consistent with its Perron vector $f$ in such a way that $f(u)>f(v)$ implies $u \prec v$ and hence $d(u) \geq d(v)$.

It is important to note that this condition is not sufficient in general. Let $\pi=$ $(4,4,3,3,2,1,1)$, then there exist two BFD-graphs but only one has greatest maximum eigenvalue, see Figure 1.
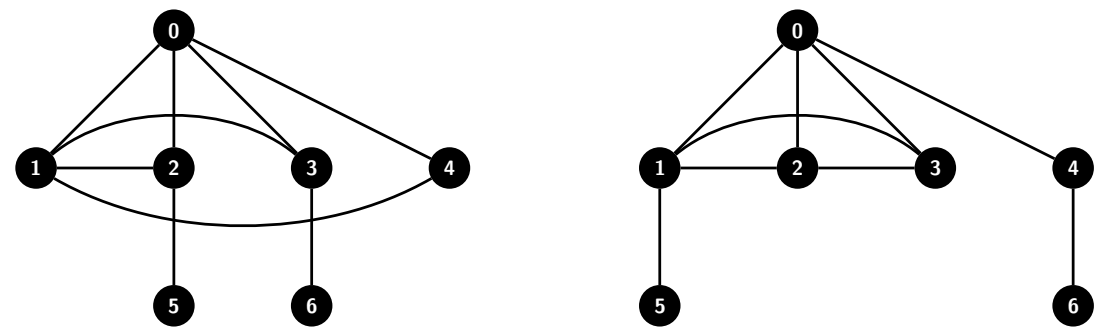

Figure 1: Two BFD-graphs with degree sequence $\pi=(4,4,3,3,2,1,1)$ that satisfy the conditions of Theorem 1.

l.h.s.: $\lambda=3.0918, f=(0.5291,0.5291,0.3823,0.3823,0.3423,0.1236,0.1236)$,

r.h.s.: $\lambda=3.1732, f=(0.5068,0.5023,0.4643,0.4643,0.1773,0.1583,0.0559)$

Trees are of special interest. Hence we are looking at the class $\mathcal{T}_{\pi}$ of all trees with given sequence $\pi$. Notice that sequences $\pi=\left(d_{0}, \ldots, d_{n-1}\right)$ is a degree sequence of a tree if and only if every $d_{i}>0$ and $\sum_{i=0}^{n-1} d_{i}=2(n-1)$, see [7]. In this class there is a single graph with BFD-ordering, see Figure 2.

Theorem 2. A tree $G$ with degree sequence $\pi$ has greatest maximum eigenvalue in class $\mathcal{T}_{\pi}$ if and only if it is a BFD-tree. $G$ is then uniquely determined up to isomorphism. The $B F D$-ordering is consistent with the Perron vector $f$ of $G$ in such a way that $f(u)>f(v)$ implies $u \prec v$.

For a tree with degree sequence $\pi$ a sharp upper bound on the largest eigenvalue can be found by computing the corresponding BFD-tree. Obviously finding this tree can be done in $O(n)$ time if the degree sequence is sorted. 


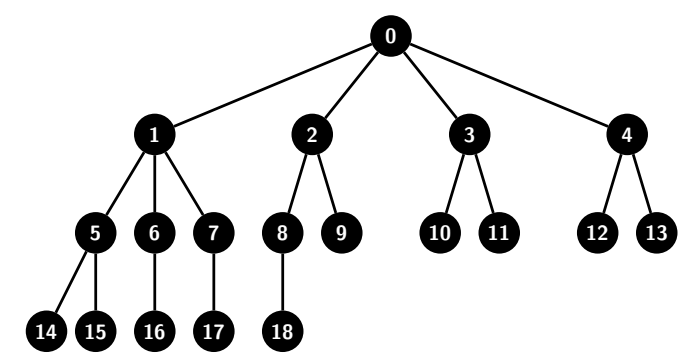

Figure 2: A BFD-tree with degree sequence $\pi=\left(4^{2}, 3^{4}, 2^{3}, 1^{10}\right)$

We define a partial ordering on degree sequences as follows: for two sequences $\pi=$ $\left(d_{0}, \ldots, d_{n-1}\right)$ and $\pi^{\prime}=\left(d_{0}^{\prime}, \ldots, d_{n-1}^{\prime}\right), \pi \neq \pi^{\prime}$, we write $\pi \triangleleft \pi^{\prime}$ if and only if $\sum_{i=0}^{j} d_{i} \leq$ $\sum_{i=0}^{j} d_{i}^{\prime}$ for all $j=0, \ldots n-1$ (recall that the degree sequences are non-increasing). Such an ordering is sometimes called majorization.

Theorem 3. Let $\pi$ and $\pi^{\prime}$ two distinct degree sequences of trees with $\pi \triangleleft \pi^{\prime}$. Let G and $G^{\prime}$ be trees with greatest maximum eigenvalues in classes $\mathcal{C}_{\pi}$ and $\mathcal{C}_{\pi^{\prime}}$, resp. Then $\lambda(G)<\lambda\left(G^{\prime}\right)$.

We get the following well-known result as an immediate corollary.

Corollary 4. A tree $G$ has greatest maximum eigenvalue in the class of all trees with $n$ vertices and $k$ leaves if and only if it is a star with paths of almost the same lengths attached to each of its $k$ leaves.

Proof. The tree sequence $\pi^{*}=(k, 2, \ldots, 2,1, \ldots, 1)$ is maximal the class of trees with $k$ pendant vertices w.r.t. ordering $\triangleleft$. Thus the statement immediately follows from Theorems 2 and 3.

\section{Proof of the Theorems}

We recall that $\lambda(G)$ denotes the maximum eigenvalue of $G$. Let $N_{f}(v)=\sum_{u v \in E} f(u)$. Thus the adjacency matrix $A(G)$ can be defined by $(A f)(v)=N_{f}(v)$. The Rayleigh quotient of the adjacency matrix $A(G)$ on vectors $f$ on $V$ is the fraction

$$
\mathcal{R}_{G}(f)=\frac{\langle A f, f\rangle}{\langle f, f\rangle}=\frac{\sum_{v \in V} f(v) \sum_{u v \in E} f(u)}{\sum_{v \in V} f(v)^{2}}=\frac{2 \sum_{u v \in E} f(u) f(v)}{\sum_{v \in V} f(v)^{2}} .
$$

By the Rayleigh-Ritz Theorem we find the following well-known property for the spectral radius of $G$.

Proposition 1 ([8]). Let $\mathcal{S}$ denote the set of unit vectors on $V$. Then

$$
\lambda(G)=\max _{f \in \mathcal{S}} \mathcal{R}_{G}(f)=2 \max _{f \in \mathcal{S}} \sum_{u v \in E} f(u) f(v) .
$$

Moreover, if $\mathcal{R}_{G}(f)=\lambda(G)$ for a (positive) function $f \in \mathcal{S}$, then $f$ is an eigenvector corresponding to the largest eigenvalue $\lambda(G)$ of $A(G)$, i.e., it is a Perron vector. 
The following technical lemma will be useful.

Lemma 2. Let $f$ be the Perron vector of a connected graph $G$. Then $f(u) \geq f(v)$ if and only if $N_{f}(u) \geq N_{f}(v)$. Moreover, for each edge $u v \in E$ where $v$ is a pendant vertex and $u$ is not, $\lambda(G)=f(u) / f(v)$ and $f(u)>f(v)$.

Proof. The first statement immediately follows from the positivity of the Perron vector and the fact that $f(v)=N_{f}(v) / \lambda$. For the second statement notice that the largest eigenvalue of a path with one interior vertex is $\sqrt{2}$. Thus the result follows by the wellknown fact that $\lambda(H) \leq \lambda(G)$ for a connected subgraph $H$ of $G$.

The main techniques for proving our theorems is rearranging of edges. We need two standard types of rearrangement steps that we call switching and shifting, respectively, in the following.

Lemma 3 (Switching $[9,14])$. Let $G(V, E)$ be a graph in class $\mathcal{C}_{\pi}$ with some edges $v_{1} u_{1}$ and $v_{2} u_{2}$. Assume that $v_{1} v_{2}, u_{1} u_{2} \notin E$. Then we get a new graph $G^{\prime}\left(V, E^{\prime}\right)$ with the same degree sequence $\pi$ by replacing $v_{1} u_{1}$ and $v_{2} u_{2}$ with edges $v_{1} v_{2}$ and $u_{1} u_{2}$ (switching). Let $f$ is a Perron vector of $G$ then we find $\lambda\left(G^{\prime}\right) \geq \lambda(G)$, whenever $f\left(v_{1}\right) \geq f\left(u_{2}\right)$ and $f\left(v_{2}\right) \geq f\left(u_{1}\right)$. The inequality is strict if and only if at least one of these two inequalities is strict.

Proof. By removing and inserting edges we obtain

$$
\begin{aligned}
\mathcal{R}_{G^{\prime}}(f)-\mathcal{R}_{G}(f) & =\left\langle A\left(G^{\prime}\right) f, f\right\rangle-\langle A(G) f, f\rangle \\
& =2\left(\sum_{x y \in E^{\prime} \backslash E} f(x) f(y)-\sum_{u v \in E \backslash E^{\prime}} f(u) f(v)\right) \\
& =2\left(f\left(v_{1}\right) f\left(v_{2}\right)+f\left(u_{1}\right) f\left(u_{2}\right)-f\left(v_{1}\right) f\left(u_{1}\right)+f\left(v_{2}\right) f\left(u_{2}\right)\right) \\
& =2\left(f\left(v_{1}\right)-f\left(u_{2}\right)\right) \cdot\left(f\left(v_{2}\right)-f\left(u_{1}\right)\right) \\
& \geq 0,
\end{aligned}
$$

and hence $\lambda\left(G^{\prime}\right) \geq \mathcal{R}_{G^{\prime}}(f) \geq \mathcal{R}_{G}(f)=\lambda(G)$ by Proposition 1. Moreover, $\lambda\left(G^{\prime}\right)=\lambda(G)$ if and only if $f$ is also an eigenvector corresponding to $\lambda\left(G^{\prime}\right)$ on $G^{\prime}$ and hence

$$
\begin{aligned}
\lambda(G) f\left(v_{1}\right) & =(A(G) f)\left(v_{1}\right)=f\left(u_{1}\right)+\sum_{w v_{1} \in E \cap E^{\prime}} f(w) \\
=\lambda\left(G^{\prime}\right) f\left(v_{1}\right) & =\left(A\left(G^{\prime}\right) f\right)\left(v_{1}\right)=f\left(v_{2}\right)+\sum_{w v_{1} \in E \cap E^{\prime}} f(w)
\end{aligned}
$$

and hence $f\left(u_{1}\right)=f\left(v_{2}\right)$. Analogously we find $f\left(v_{1}\right)=f\left(u_{2}\right)$.

Lemma 4 (Shifting $[1,2]$ ). Let $G(V, E)$ be a graph in class $\mathcal{C}_{\pi}$, and let $u v_{1} \in E$ and $u v_{2} \notin E$. Then we get a new graph $G^{\prime}\left(V, E^{\prime}\right)$ by replacing edge uv $v_{1}$ by the edge uv $v_{2}$ (shifting). Let $f$ is a Perron vector of $G$ then we find $\lambda\left(G^{\prime}\right)>\lambda(G)$, whenever $f\left(v_{2}\right) \geq$ $f\left(v_{1}\right)$. 
Proof. Analogously to the proof of Lemma 3 we find $\lambda\left(G^{\prime}\right) \geq \mathcal{R}_{G^{\prime}}(f) \geq \mathcal{R}_{G}(f)=\lambda(G)$. If equality holded then $f$ would also be a Perron vector of $G^{\prime}$ and thus $\lambda\left(G^{\prime}\right) f\left(v_{2}\right)=$ $\sum_{x v_{2} \in E} f(x)+\sum_{y v \in E^{\prime} \backslash E} f(y)>\sum_{x v_{2} \in E} f(x)=\lambda(G) f\left(v_{2}\right)$, a contradiction.

Lemma 5. Let $f$ be the Perron vector of a graph $G$ in $\mathcal{C}_{\pi}$. Let $u$ and $v$ be two vertices with $d(u)>d(v)$. If $f(u)<f(v)$ then $G$ cannot have greatest maximum eigenvalue in $\mathcal{C}_{\pi}$.

Proof. Let $d(u)-d(v)=c>0$ and assume $f(u)<f(v)$. Then there are (at least) $c$ neighbors $w_{k}$ of $u$ that are not adjacent to $v$. When we replace these edges $w_{1} u, \ldots, w_{c} u$ by the edges $w_{1} v, \ldots, w_{c} v$ we get a new graph $G^{\prime}$ with the same degree sequence $\pi$. The neighbors $c$ can be chosen such that $G^{\prime}$ remains connected, since either $u$ and $v$ have a common neighbor or are adjacent, or we can select any of the neighbors of $u$. By Lemma 4 we then have $\lambda\left(G^{\prime}\right)>\lambda(G)$ and the statement follows.

Lemma 6. Let $f$ be the Perron vector of a graph $G$ in $\mathcal{C}_{\pi}$. Let $v u \in E(G)$ and $v x \notin E(G)$ with $f(u)<f(x) \leq f(v)$. If $f(v) \geq f(w)$ for all neigbors $w$ of $x$, then $G$ cannot have greatest maximum eigenvalue in $\mathcal{C}_{\pi}$.

Proof. Assume that such vertices exist. Construct a new graph $G^{\prime}\left(V, E^{\prime}\right)$ with the same degree sequence $\pi$ by replacing edges $v u$ and $x w$ by edges $v x$ and $u w$. Then by Lemma 3, $\mathcal{R}_{G^{\prime}}(f)>\mathcal{R}_{G}(f)$. It remains to show that we can choose vertex $w$ such that $G^{\prime}$ is connected. Then $G^{\prime} \in \mathcal{C}_{\pi}$ and hence $G$ cannot have the greatest maximum eigenvalue.

First, notice that there must be a neighbor $p$ of $x$ that is not adjacent to $u$, since otherwise $N_{f}(x)=\sum_{w x \in E} f(w) \leq \sum_{y u \in E} f(y)=N_{f}(u)$ and thus by Lemma $2, f(x) \leq f(u)$, a contradiction to our assumptions. Furthermore, $x$ must have at least two neighbors, since otherwise we had by Lemma 2 and assumption $f(x)>f(u), f(w)=N_{f}(x)>$ $N_{f}(u) \geq f(v)$, a contradiction to $f(w) \leq f(v)$. Since $G$ is connected there is a simple path $P_{v x}=(v, \ldots, t, x)$ from $v$ to $x$. Then there are four cases:

(1) If $v u \notin P_{v x}$ and $u t \notin E(G)$, then we set $w=t$.

(2) Else, if $v u \notin P_{v x}$ and $u t \in E(G)$, then we set $w$ to one of the neighbors of $x$ that are not adjacent to $u$.

(3) Else, if $v u \in P_{v x}$ and all neighbors not equal $t$ are adjacent to $u$. Then $t$ cannot be adjacent to $u$ and we set $w=t$.

(4) Else, $v u \in P_{v x}$ and there exists a neighbor $p$ of $x, p \neq t$, with $u p \notin E(G)$. Then we set $w=p$.

In either case $G^{\prime}$ remains connected. Thus the statement follows.

Proof of Theorem 1. Assume that $G(V, E)$ has greatest maximum eigenvalue in class $\mathcal{C}_{\pi}$. Let $f$ be a Perron vector of $G$. Create an ordering $\prec$ by breadth-first search as follows: Choose the maximum of $f$ as root $v_{0}$ in layer 0 ; append all neighbors $v_{1}, \ldots, v_{d\left(v_{0}\right)}$ of $v_{0}$ to the list ordered list; these neighbors are ordered such that $u \prec v$ whenever $d(u)>d(v)$, or $d(u)=d(v)$ and $f(u)>f(v)$ (in the remaining case the ordering can be arbitrary); 
then continue recursively with all vertices $v_{1}, v_{2}, \ldots$ until all vertices of $G$ are processed. Notice that (B1) holds for this ordering.

We first show that $u \prec v$ implies $f(u) \geq f(v)$ for all $u, v \in V$. Suppose there exist two vertices $v_{i}$ and $v_{j}$ with $v_{i} \prec v_{j}$ but $f\left(v_{i}\right)<f\left(v_{j}\right)$. Notice that $v_{i}$ cannot be root $v_{0}$. Let $w_{i}$ and $w_{j}$ be the parents of $v_{i}$ and $v_{j}$, respectively. By construction there are two cases: (i) $w_{i}=w_{j}$, or (ii) $w_{i} \prec w_{j}$. For case (i) we have $d\left(v_{i}\right) \geq d\left(v_{j}\right)$ by construction and $d\left(v_{i}\right) \leq d\left(v_{j}\right)$ by Lemma 5 and thus $d\left(v_{i}\right)=d\left(v_{j}\right)$. But then we had $v_{i} \succ v_{j}$ by the definition of our ordering, since $f\left(v_{i}\right)<f\left(v_{j}\right)$, a contradiction.

For case (ii) assume that $v_{j}$ is maximal, i.e., for any other vertex $u$ with this property we have $f(u) \leq f\left(v_{j}\right)$. Let $v_{i}\left(\prec v_{j}\right)$ be the first vertex (in the ordering of $\prec$ ) with $f\left(v_{i}\right)<f\left(v_{j}\right)$. Hence $f(u) \geq f\left(v_{j}\right)$ for each $u \prec v_{i}$ and we find $f\left(w_{i}\right) \geq f\left(v_{j}\right)>f\left(v_{i}\right)$. Note that $v_{j}$ cannot be adjacent neither to $w_{i}$ nor to $v_{0}$ as we then had case (i). Thus $f\left(w_{i}\right) \geq f\left(u_{j}\right)$ for all neighbors $u_{j}$ of $v_{j}$, since otherwise $v_{j}$ were not maximal. Hence $G$ can not have greatest maximum eigenvalue by Lemma 6 , a contradiction. At last we have to show Property (B2). However, this follows immediately from Lemma 5.

Proof of Theorem 2. The necessity condition is an immediate corollary of Theorem 1. To show that two BFD-trees $G$ and $G^{\prime}$ in class $\mathcal{T}_{\pi}$ are isomorphic we use a function $\phi$ that maps the vertex $v_{i}$ in the $i$-th position in the BFD-ordering of $G$ to the vertex $w_{i}$ in the $i$-th position in the BFD-ordering of $G^{\prime}$. By the properties (B1) and (B2) $\phi$ is an isomorphism, as $v_{i}$ and $w_{i}$ have the same degree and the images of neighbors of $v_{i}$ in the next layer are exactly the neigbors of $w_{i}$ in the next layer. The latter can be seen by looking on all vertices of $G$ in the reverse BFD-ordering. Thus the proposition follows.

Proof of Theorem 3. Let $\pi=\left(d_{0}, \ldots, d_{n-1}\right)$ and $\pi^{\prime}=\left(d_{0}^{\prime}, \ldots, d_{n-1}^{\prime}\right)$ be two non-increasing tree sequences with $\pi \triangleleft \pi^{\prime}$, i.e., $\pi \neq \pi^{\prime}, \sum_{i=0}^{j} d_{i} \leq \sum_{i=0}^{j} d_{i}^{\prime}$, and $\sum_{i=0}^{n-1} d_{i}=\sum_{i=0}^{n-1} d_{i}^{\prime}=$ $2(n-1)$. Let $G$ have greatest maximum eigenvalue in $\mathcal{T}_{\pi}$. By Theorem $2 G$ has a BFDordering that is consistent with $f$, i.e., $f(u)>f(v)$ implies $u \prec v$.

First assume that $\pi$ and $\pi^{\prime}$ differ only in two positions $k$ and $l$ with $d_{k}^{\prime}=d_{k}+1$ and $d_{l}^{\prime}=d_{l}-1$ (and hence $k<l$ and $d_{k} \geq d_{l}>1$ ). Let $v_{k}$ and $v_{l}$ be the corresponding vertices in $G$. Without loss of generality we assume that $f\left(v_{k}\right) \geq f\left(v_{l}\right)$. Since $G$ is a tree and $d\left(v_{l}\right) \geq 2$, there exists a neighbor $w$ of $v_{l}$ in layer $h\left(v_{l}\right)+1$ that is not adjacent to $v_{k}$. Thus we can shift edge $v_{l} w$ by $v_{k} w$ and get a new tree $G^{\prime}$ with degree sequence $\pi^{\prime}$ and $\lambda\left(G^{\prime}\right)>\lambda(G)$ by Lemma 4 .

For two tree sequences $\pi \triangleleft \pi^{\prime}$ we can find a sequence of tree sequences $\pi=\pi_{0} \triangleleft \pi_{1} \triangleleft \cdots \triangleleft$ $\pi_{k}=\pi^{\prime}$ where $\pi_{i-1}$ and $\pi_{i}(i=1, \ldots, n)$ differ only in two positions as described above by the following recursive procedure. For $\pi_{i-1}$ let $j$ be the first position in which $\pi_{i-1}$ and $\pi^{\prime}$ differ. Then $d_{j}^{(i-1)}<d_{j}^{\prime}$ and we construct $\pi_{i}=\left(d_{0}^{(i)}, \ldots, d_{n-1}^{(i)}\right)$ by $d_{j}^{(i)}=d_{j}^{(i-1)}+1$, $d_{j+1}^{(i)}=d_{j+1}^{(i-1)}-1$, and $d_{l}^{(i)}=d_{l}^{(i-1)}$ otherwise. If necessary, $\pi_{i}$ is then sorted nonincreasingly. Thus $\pi_{i}$ again is a tree sequence and the statement follows. 


\section{Remarks}

In general, we can ask the same questions for Perron vectors of generalized graph Laplacians, i.e., symmetric matrices with non-positive off-diagonal entries. In this paper we showed that switching and shifting operations are compatible with respect to degree sequences and we used them to find trees or connected graphs with greatest maximum eigenvalue of the adjacency matrix. In [2] these operations were applied to construct graphs with the smallest first eigenvalue of the so called Dirichlet matrix. Here the corresponding minization problems are called Faber-Krahn-type inequalities. We refer the interested reader to [3] and the references given therein.

One also might ask whether one can find the smallest maximum eigenvalue in a class $\mathcal{C}_{\pi}$ by the same procedure. It is possible to apply shifting in the proof of Theorem 1 just the "other way round". We then would arrive at trees that are constructed by breadthfirst search but with increasing vertex degrees for non-pendant vertices. However, this idea does not work. Figure 3 shows a counterexample.
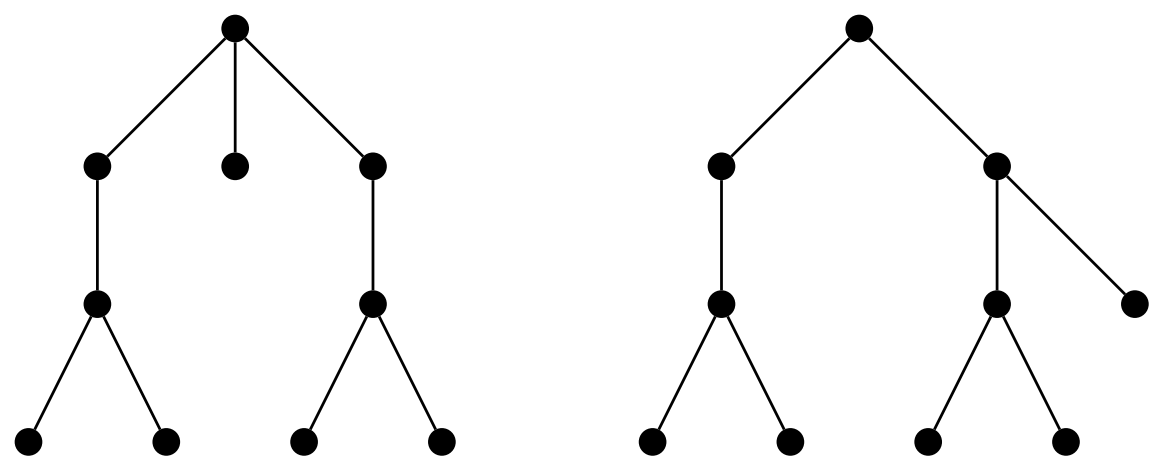

Figure 3: Two trees with degree sequence $(2,2,3,3,3,1,1,1,1,1)$. The tree on the l.h.s. has smallest maximum eigenvalue $(\lambda=2.1010)$ among all trees in $\mathcal{C}_{\pi}$. The tree on the r.h.s. has a breadth-first ordering of the vertices with increasing degree sequences (and thus has lowest first Dirichlet eigenvalue). However it does not minimize the maximum eigenvalue $(\lambda=2.1067)$

\section{Acknowledgment}

The authors would like to thank Christian Bey for calling our attention to eigenvalues of the adjacency matrix of a graph. We thank Gordon Royle and Brendan McKay for their databases of combinatorial data on graphs. This was of great help to find the two counterexamples in Figures 1 and 3. We also thank the Institute for Bioinformatics of the University in Leipzig for the hospitality and for providing a scientific working environment while we wrote down this paper. The first author is partially supported by the Belgian Programme on Interuniversity Attraction Poles, initiated by the Belgian Federal Science Policy Office, and a grant Action de Recherche Concertée (ARC) of the Communauté Française de Belgique. 


\section{References}

[1] F. Belardo, E. M. L. Marzi, and S. K. Simić. Some results on the index of unicyclic graphs. Linear Algebra Appl., 416(2-3):1048-1059, 2006.

[2] T. Bıyıkoğlu and J. Leydold. Faber-Krahn type inequalities for trees. J. Comb. Theory, Ser. B, 97(2):159-174, 2006.

[3] T. Bıyıkoğlu, J. Leydold, and P. F. Stadler. Laplacian Eigenvectors of Graphs. Perron-Frobenius and Faber-Krahn Type Theorems, volume 1915 of Lecture Notes in Mathematics. Springer, 2007.

[4] R. A. Brualdi and E. S. Solheid. On the spectral radius of connected graphs. Publ. Inst. Math. (Beograd), 39(53):45-54, 1986.

[5] D. Cvetkovic and P. Rowlinson. The largest eigenvalue of a graph: A survey. Linear Multilinear Algebra, 28(1/2):3-33, 1990.

[6] D. M. Cvetković, P. Rowlinson, and S. Simić. Eigenspaces of Graphs, volume 66 of Encyclopdia of Mathematics and its Applications. Cambrigdge University Press, Cambridge, UK, 1997.

[7] J. Edmonds. Existence of $k$-edge connected ordinary graphs with prescribed degrees. J. Res. Nat. Bur. Standards Sect. B, 68B:73-74, 1964.

[8] R. A. Horn and C. R. Johnson. Matrix Analysis. Reprinted with corrections. Cambridge University Press, 1990.

[9] J. Leydold. A Faber-Krahn-type inequality for regular trees. GAFA, Geom. Funct. Anal., 7(2):364-378, 1997.

[10] B. Liu, J. Shen, and X. Wang. On the largest eigenvalue of non-regular graphs. J. Comb. Theory, Ser. B, 97(6):1010-1018, 2007. doi: 10.1016/j.jctb.2007.02.008.

[11] O. Melnikov, R. I. Tyshkevich, V. A. Yemelichev, and V. I. Sarvanov. Lectures on Graph Theory. B.I. Wissenschaftsverlag, Mannheim, 1994. Transl. from the Russian by N. Korneenko with the collab. of the authors.

[12] A. R. Pruss. Discrete convolution-rearrangement inequalities and the Faber-Krahn inequality on regular trees. Duke Math. J., 91(3):463-514, 1998.

[13] O. Rojo. The spectra of some trees and bounds for the largest eigenvalue of any tree. Linear Algebra Appl., 414(1):199-217, 2006.

[14] P. Rowlinson. Graph perturbations. In Surveys in Combinatorics, Proc. 13th Br. Comb. Conf. Guildford/UK 1991. Lond. Math. Soc., 1991.

[15] J. Shu and Y. Wu. Sharp upper bounds on the spectral radius of graphs. Linear Algebra Appl., 377:241-248, 2004.

[16] S. Simić, E. M. L. Marzi, and B. Francesco. On the index of caterpillars. Discrete Math., 308(2-3):324-330, 2008. doi: 10.1016/j.disc.2006.11.046. 